\title{
Controlling Binder Adhesion to Impact Electrode Mesostructure and Transport
}

\author{
Ishan Srivastava, ${ }^{*}$ Dan S. Bolintineanu, Jeremy B. Lechman, and Scott A. \\ Roberts*
}

Sandia National Laboratories, Albuquerque, New Mexico 87185 USA

E-mail: isriva@sandia.gov; sarober@sandia.gov

\section{Abstract}

The complex three-phase composition of lithium-ion battery electrodes - containing an ion-conducting pore phase, a nanoporous electron-conducting carbon binder domain (CBD) phase, and an active material (AM) phase - provides several avenues of mesostructural engineering to enhance battery performance. We demonstrate a promising strategy for engineering electrode mesostructures by controlling the strength of adhesion between the AM and CBD phases. Using high-fidelity, physics-based colloidal and granular dynamics simulations, we predict that this strategy can provide significant control over electrochemical transport-relevant properties such as ionic conductivity, electronic conductivity, and available AM surface area. Importantly, the proposed strategy could be experimentally realized through surface functionalization of the $\mathrm{AM}$ and $\mathrm{CBD}$ phases and would be compatible with traditional electrode manufacturing methods.

\section{Keywords}

Li-ion battery; mesoscale electrode modeling; carbon binder domain; adhesion; cohesion; granular materials; colloidal dynamics; effective transport properties

\section{Introduction}

Lithium-ion batteries (LIB) are the leading energy storage technology and are everincreasingly adopted in electric vehicles and personal electronics. ${ }^{1}$ Although LIBs have higher energy density and lower cost compared to other battery systems, there is a persistent demand for even higher energy density. Significant research towards this goal is focused on developing new material chemistries that intrinsically offer high energy density. ${ }^{2}$ However, a more tractable approach involves mesostructural engineering of existing Li-ion material chemistries. Several approaches have been considered towards this goal, such as fabricating denser and/or thicker electrodes, ${ }^{3}$ controlling electrode topology by fabricating ordered porous structures ${ }^{4}$ engineering hierarchical pore size distributions, ${ }^{5}$ and inducing pore space alignment. ${ }^{6}$ The obvious approach of increasing electrode thickness and/or density guarantees higher energy density; however, it often results in poorer power density due to restricted ionic transport. ${ }^{7}$ Other approaches involving pore space engineering produce low ionic tortuosity electrodes with high energy density; however, the manufacturing methods involved in their fabrication are expensive and often not compatible with conventional slurrybased LIB manufacturing methods.

LIB electrodes contain four dominant materials: (1) active material (AM) typically composed of micron-sized particles of an electro- 
active material; (2) electron-conducting carbon black nanoparticles; ${ }^{8}$ (3) polymeric binder that provides mechanical strength to the electrode; and (4) ion-conducting electrolyte that infiltrates a topologically tortuous pore space. Recent experiments have indicated that carbon black nanoparticles and polymeric binder aggregate during electrode manufacturing into a nanoporous carbon binder domain (CBD) phase with a typical length scale of $100 \mathrm{~nm} .{ }^{8-11}$ The industrial battery manufacturing process involves mixing AM particles, carbon black nanoparticles, and polymeric binder into a nonaqueous solvent (such as N-methyl pyrrolidone) to form a slurry, which is then coated onto a current collector, dried to remove the solvent, and calendered into high density electrodes, which are eventually filled with a liquid electrolyte. ${ }^{12}$ The slurry-based manufacturing process is low-cost and highly scalable; as a result, it is the method of choice for large-scale industrial battery manufacturing. Therefore, any technique that enables tailoring electrode mesostructure to enhance its electrochemical performance - and is also compatible with conventional slurry-based manufacturing processes — would be significantly impactful.

In this paper, we demonstrate a potential route for controlling electrode mesostructure and its electrochemical performance through simultaneous control of two key material parameters in the conventional battery manufacturing process: cohesion within the CBD phase and adhesion between AM particles and the CBD phase. We recognize that the precursor slurry of electrode material is composed of large micron-sized AM particles that are suspended in a carbon black and binder colloid. ${ }^{8}$ Using physics-based granular and colloidal modeling techniques on a large scale, we simulate slurry fabrication, drying, and eventual calendering into low-porosity electrodes. We elucidate the role of AM-CBD adhesion and CBD-CBD cohesion on the electrode mesostructure and key transport-relevant properties. In practice, cohesion and adhesion between AM and CBD phases can be controlled by either adding depletants, ${ }^{13}$ modifying the solvent salt concentration, ${ }^{14}$ or by modifying particle surface rough- ness which has the equivalent effect of modifying the effective attraction between two materials. ${ }^{15}$ Such techniques for controlling cohesion and adhesion in granular and colloidal materials are well-described in the literature, ${ }^{16}$ and could be adapted to battery manufacturing. Several prior studies have reported chemical modifications to the binder for increased binder adhesion with the active material that result in improved battery cyclability. ${ }^{17}$ This study focuses on the effect of cohesion and adhesion on electrode mesostructure and electrochemical transport.

Imaging the material phases within a battery electrode is a challenging task and requires a combination of techniques to reliably reproduce the electrode mesostructure. Significant advances in X-ray micro-tomography (XCT) have revealed a particulate nature of the AM phase along with its spatial arrangement within an electrode. ${ }^{9,18-21}$ However, XCT can not easily differentiate the pore and CBD phases, as both of these phases are relatively transparent to the high-energy X-rays used for AM phase identification. Advanced imaging techniques such as the combination of focused ion beam (FIB) milling and scanning electron microscopy $(\mathrm{SEM})^{10,19,20,22}$ can be used in conjunction with $\mathrm{XCT}$ to resolve the CBD and pore phases at the nanoscale. Recently, another technique involving infiltrating the CBD with iron nanoparticles was used in conjunction with $\mathrm{XCT}$ to resolve CBD and pore phase at the nanoscale. ${ }^{11}$ However, the imaged sample volume in these techniques is small and is not descriptive of their topological arrangement within the electrode. Additionally, the FIB-SEM technique is destructive and can alter the mesostructure in unpredictable ways.

Consequently, several computational techniques have been developed to reconstruct the pore and CBD phases around the experimentally-imaged AM phase. ${ }^{9,23-26}$ However, these techniques lack a strong physical basis and do not provide any prediction of electrode mesostructure based on material properties of its constituents, even though they predict the sensitivity of electrochemical properties on CBD morphology. ${ }^{9,23,24}$ Alternatively, 
particle-based methods such as discrete element method, ${ }^{27-29}$ and coarse-grained molecular dynamics $^{30-34}$ with parameterized force fields ${ }^{35}$ have been used to simulate the actual physics of electrode processing and provide predictive correlations between electrode mesostructure and material properties. However, these methods are computationally expensive; ${ }^{36,37}$ as a result, they have predominantly been applied to idealized electrode materials of small sizes.

The novelty of simulation methods and enclosed results in this paper are: (i) we have simulated the fabrication (i.e., drying and calendering) of LIB electrodes at experimentallyrelevant length scales of $100 \mu \mathrm{m}$; (ii) we have used realistic size distribution of AM particles obtained from experimental data and experimentally-relevant weight ratios of AM and CBD phases in the simulations; (iii) using a combination of granular and colloidal simulations along with finite element analyses, we have described a robust computational framework for studying process-structure-property relationships in LIB electrodes in a physically meaningful way; and (iv) by systematically varying the strength of CBD cohesion and AMCBD adhesion, we have highlighted the remarkable effects of controlling these material parameters on the mesostructure and transport properties of LIB electrodes. We demonstrate that our predictions of transport properties compare well with previously reported experiments ${ }^{38}$ and computer-generated electrode mesostructures with a reconstructed CBD phase, ${ }^{23,24}$ thus implicating a strong dependence of transport properties on the CBD morphology.

\section{Methods}

\subsection{Electrode Dynamics and Composition}

Here we provide a brief description of the colloidal and granular dynamics simulations employed in this study. The reader is directed to the Supporting Information for a more detailed description. The AM of interest is 2500 poly-disperse, spherical particles, whose size distribution is matched to the experimentally measured particle size distribution reported by Ebner et al. ${ }^{18}$ for $\mathrm{LiNi}_{1 / 3} \mathrm{Mn}_{1 / 3} \mathrm{Co}_{1 / 3} \mathrm{O}_{2}$ (NMC333) particles and is restricted to a minimum and maximum diameter of 2 and $20 \mu \mathrm{m}$ respectively. The CBD phase within the electrode is represented by uniformly sized coarsegrained spheres of $500 \mathrm{~nm}$ diameter, where each sphere is assumed to be composed of $50 \%$ polyvinylidene fluoride (PVDF) binder and $50 \%$ carbon black by weight. Our choice of CBD diameter is supported by experimental imaging of the CBD, ${ }^{10}$ and rheological measurements of CBD in LIB slurry. ${ }^{8}$ Four different weight loading ratios of $\mathrm{AM}$ :CBD ranging from 90:10 to 96:4 were analyzed,resulting in approximately one to two million CBD spheres for the 2500 AM spheres in each simulation. The large number of coarse-grained CBD particles employed in this study provide a nearly-continuum representation of the CBD phase within the electrode, thus enabling a high-fidelity analysis of electrode mesostructure and electrochemical transport.

The AM and CBD spheres are assumed to be suspended in an implicit background solvent that provides a mean-field viscous drag to the particle motion. Any long-ranged hydrodynamics effects of the solvent are ignored. A recent study has highlighted that long-ranged hydrodynamics play a negligible role in the structure of dense colloids, ${ }^{39}$ which is the case here.

The AM particles - as a result of their large size - are treated as non-Brownian, elastic and frictional granular spheres that are suspended within the solvent; as such, their interactions upon contact are suitably described by the Hertz-Mindlin frictional contact mechanics model. ${ }^{40}$ The sub-micron sized CBD particles are colloidal in nature, and they can diffuse through the solvent by Brownian motion with a diffusivity that depends on their size and the solvent viscosity. The interaction between two contacting CBD particles are described by the cohesive Johnson-Kendall-Roberts (JKR) contact mechanics model, ${ }^{41}$ with a tunable strength of cohesive surface energy that is an important ingredient in the present simulations. Similarly, the interaction between a CBD 
and an AM particle is also described by the JKR contact mechanics model, with a tunable strength of adhesive surface energy. Although the JKR contact mechanics model only approximately predicts the interfacial stress of two contacting spheres with prescribed surface energies, the analytical form of this model ${ }^{41}$ is computationally efficient for a particle-based simulation method, and has been used and validated previously in simulations of solvent-free battery electrodes. ${ }^{29}$ In all the results that follow, the strength of cohesion and adhesion is normalized by the thermal energy $k T$ of the system. The reader is directed to the Supporting Information for a detailed description of JKR contact mechanics model.

The motion of the AM and CBD particles within the solvent is simulated using a combination of discrete element and colloidal dynamics methods that resolves particle trajectories in time via explicit time integration of the equations of motion. The open-source molecular dynamics software LAMMPS is used to simulate colloidal and granular dynamics. ${ }^{42}$ The reader is directed to the Supporting Information for a detailed description of the particles' equations of motion.

A detailed micromechanical description of interfacial contacts between AM and CBD phases is unknown, with the exception of nanoindentation studies that have characterized the effective mechanical strength of the LIB electrodes $^{43}$ and surface tension measurements of the surface energy of various component of an electrode. ${ }^{43}$ While a particle-based representation of the AM phase is reasonable, a particlebased representation of the CBD phase and the assumption of Hertz-Mindlin and JKR contact mechanics between these phases is approximate. However, this study demonstrates that the mesostructure and transport properties of LIB electrodes is strongly related to the micromechanics of AM and CBD phases, and the simulations described here explore these relationships in a physically meaningful way.

\subsection{Electrode Fabrication Simu- lations}

Initially, the AM and CBD particles are randomly dispersed in the solvent at a total solid volume fraction of $10 \%$, as shown in Figure 1 (Ia), representing the initial state of the electrode. The simulation box is periodic in all directions, with lateral dimensions of 110 $\mu \mathrm{m}$ and an initial height of $\sim 700 \mu \mathrm{m}$. The first stage of electrode fabrication, slurry drying, is simulated by compressing the simulation box from the top - while the lateral dimensions are kept unchanged - until the system is densified to a total solid volume fraction of $50 \%$, as shown in Figure 1(Ib). The box compression effectively represents solvent drying from the top surface of the slurry, while neglecting any evaporation-induced hydrodynamics. The compression is performed sufficiently slowly (within computational limits) to enable substantial diffusion of the CBD particles within the slurry. While the rate of solvent drying has been shown to significantly impact the electrode mesostructure, ${ }^{44,45}$ an examination of these effects is beyond the scope of this study. Furthermore, in slurry processing of electrodes, it has been demonstrated that only a fraction of the dissolved binder adsorbs on the active material particle surface. ${ }^{46}$ In this study, we assume that all of the binder agglomerates with carbon nanoparticles in the slurry to form the CBD.

At the end of drying, the solvent is assumed to have completely evaporated, and any further diffusion of CBD particles is prohibited by removing all solvent effects. The next stage of the simulation corresponds to electrode calendering, in which the dried $50 \%$ porous electrode is uniaxially compressed at a constant strain rate to a final porosity of $30 \%$, while the lateral dimensions are maintained at $110 \mu \mathrm{m}$, as shown in Figure 1(Ic). Upon calendering, the electrode thickness along the direction of compressionwhich is also the direction of electrochemical transport-varies from $\sim 80$ to $90 \mu \mathrm{m}$ depending on the $\mathrm{AM}$ weight loading. Although at high levels of calendering compression, the active material particles have been demonstrated to fracture into smaller constituent particles, ${ }^{47}$ 



Figure 1: Simulation methodology: (I) (a-c) The manufacturing of three-phase electrodes, from slurry drying to calendering, is simulated using granular and colloidal dynamics. The AM particles (red) are poly-disperse granular particles, and the CBD phase is represented by coarse-grained colloidal particles (blue) of a uniform size. Cross sections in the middle of the electrode are displayed after slurry drying and calendering. The white region around AM and CBD particles represents the pore space. (II) The simulated electrode in (I) is first swelled to introduce $50 \%$ nanoporosity, and then mapped onto a regular lattice to provide a spatial distribution of AM (red), CBD (blue), and pore (white) phases. (III) The effective transport properties are calculated using finite element analyses, with the spatial distribution of current density shown. All images are from the same mesostructure realization. 
a recent study highlighted that such fracture occurs when the electrodes are calendered to less than $30 \%$ porosity. ${ }^{48}$ Because the lowest porosity considered in this study is $30 \%$, we do not simulate AM particle breakage. Furthermore, calendering has also been demonstrated to induce morphological changes of the adsorbed binder depending on the binder chemistry. ${ }^{49}$ Such effects are also ignored in the present study.

\subsection{CBD Swelling for Nanoporos- ity}

Prior imaging of LIB electrodes using a combination of FIB-SEM and XCT techniques has demonstrated a two-scale hierarchy of the electrolyte-filled pore space. ${ }^{10,50}$ At the micron scale, LIB electrodes are porous. The present simulations naturally account for such microscale porosity. However, FIB-SEM imaging has shown that CBD is also $\sim 50 \%$ porous with pores of tens of nanometers in size. ${ }^{10,51}$ Additionally, recent experiments on CBD thin films demonstrated that the CBD mesostructure contained $\sim 50 \%$ porous regions. ${ }^{50}$ In the present simulations, we account for this nanoporosity by swelling the CBD particles, such that the volume occupied by the CBD phase is doubled. ${ }^{24}$ The CBD swelling is achieved by levelset methods that also modifies the discrete sphere-like nature of the CBD phase into a more homogeneous morphology, thus providing further resemblance to the imaged CBD phase in experiments. The coarse-grained swelled CBD phase is assigned an intrinsic nanoporosity of $50 \%$. Figure $1(\mathrm{II})$ depicts an image of the electrode in Figure 1(Ic) after CBD swelling.

\subsection{Transport Calculations}

LIB electrode mesostructures after CBD expansion are analyzed for their geometric morphological characteristics and transport-relevant properties. For the former, the three-phase mesostructure is mapped onto a regular lattice with a resolution of $100 \mathrm{~nm}$ per grid point, as shown in Figure 1(II). For transport analyses, the mesostructure is discretized in an un- structured mesh using the conformal decomposition finite element method, ${ }^{52}$ and the electronic and ionic transport conservation equations are solved to extract effective ionic tortuosity and electronic conductivity. A representative finite element solution depicting local current density obtained upon solving the electronic transport conservation equation on the same mesostructure in Figure 1(II) is shown in Figure 1(III). The reader is referred to Supporting Information for further details on these calculation methods.

\section{Results}

We have simulated electrode fabrication and analyzed their mesostructural and transport properties for four ratios of $\mathrm{AM}$ and $\mathrm{CBD}$ weight loading, four cohesion strengths of the CBD phase, four adhesion strengths between $\mathrm{AM}$ and $\mathrm{CBD}$ phase, and five different porosities. For each set of material parameters, three different realizations were simulated to analyze statistical variations in mesostructural and transport properties. Table 1 describes the parameter space explored in this study. Although the main text includes results for the highest and lowest ratio of AM and CBD weight loading, we have included the data from mesostructural and transport analyses for intermediate weight loading ratios in Table S1 in the Supporting Information.

\subsection{Electrode Morphology}

CBD cohesion and AM-CBD adhesion play a crucial role in the morphology of the electrode. The four panels in Figure 2 depict the cross sections in the middle of four electrodes with the same AM weight loading (90\%) and porosity

Table 1: Parameter space explored in this study.

\begin{tabular}{|c|c|}
\hline Property & Values \\
\hline AM:CBD weight loading ratio & $90: 10,92: 8,94: 6,96: 4$ \\
\hline CBD cohesion strength $[k T]$ & $0.1,1,10,100$ \\
\hline AM-CBD adhesion strength $[k T]$ & $0.1,1,10,100$ \\
\hline Electrode Porosity $[\%]$ & $50,45,40,35,30$ \\
\hline
\end{tabular}


(50\%) after solvent drying, but with different strengths of cohesion and adhesion. Although a direct comparison of the simulated mesostructures with real electrodes is not possible due to experimental challenges in binder imaging at the length scales of interest, the reader is referred to previous studies ${ }^{9,23,24}$ for comparison with hybrid experimental and synthetically generated electrode mesostructures containing both AM and CBD phases.

When CBD cohesion and AM-CBD adhesion is small, the CBD and pore phases are homogeneously well-dispersed around the AM particles (see panel (c)), and there is no well-defined structure of the CBD phase. Upon increasing CBD cohesion, while keeping the AM-CBD cohesion small, the CBD morphology becomes more connected at long distances, possessing a string-like rather than a spatially-uniform structure (see panel (d)). Such string-like aggregates have also been previously demonstrated in experiments on dense attractive colloids. ${ }^{53}$

AM-CBD adhesion has an even more dramatic effect on the electrode morphology. When CBD cohesion is low but AM-CBD adhesion is high, large volumes of CBD diffuses towards and sticks on the surface of AM particles, thus providing a uniform CBD coating around AM particles and creating large porous regions within the electrode (see panel (a)). However, this effect is somewhat mitigated when the CBD cohesion is also equivalently high. In this scenario, the diffusion of CBD towards AM surface is hindered by large CBD cohesion which creates a gel-like structurally strong CBD network within the pore space, thus preventing any further CBD migration (see panel (b)). As a result, the electrode mesostructure bears a closer resemblance to the case where CBD cohesion is high but the AM-CBD adhesion is low. The competing effects of CBD cohesion and AM-CBD adhesion on the mesostructure of the electrodes provides a powerful knob to design CBD morphology within the electrodes. In the next sections, we describe their effects on electrode transport properties.

The effects of CBD cohesion and AM-CBD adhesion are apparent even in electrodes with

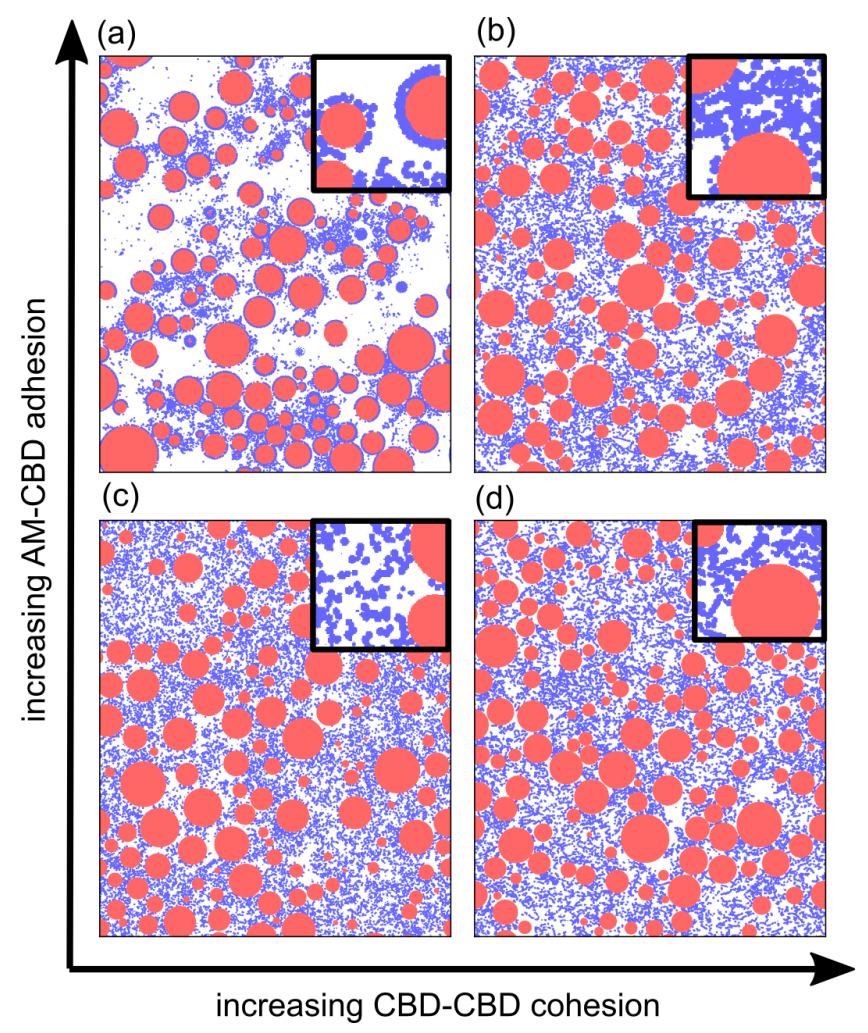

Figure 2: Cross sections $(130 \mathrm{x} 110 \mu \mathrm{m})$ of simulated electrode structures with 90\% AM weight loading and a porosity of 50\%. The AM, CBD, and pore phases are colored as red, blue, and white respectively. The four images correspond to the highest $(100 k T)$ and lowest $(0.1 k T)$ values of AM-CBD adhesion and the highest (100 $k T)$ and lowest $(0.1 k T)$ values of CBD cohesion used in the simulations. Zoomed-in images of CBD morphology around AM particles is displayed within black squares at the top corner in $(\mathrm{a})-(\mathrm{d})$.

higher AM weight loadings containing lower fractions of the CBD (see Supporting Information for cross sections of simulated electrode structures with 96\% AM weight loading). Particularly, when the AM-CBD adhesion is high and CBD cohesion is low, most of the CBD diffuses towards the AM surface, resulting in very small fractions of the CBD within the pore phase. The effect of increasing CBD cohesion, however, is not as strong as in $90 \%$ AM weight loading, because the low fraction of the CBD phase is unable to form long-ranged gel-like connected mesostructure. Consequently, the CBD aggregates into disconnected string-like morphology that are oriented orthogonal to the di- 
rection of slurry drying.

Upon calendering the electrodes towards lower porosities, the effect of CBD cohesion on the electrode mesostructure is reduced, as the CBD phase is compressed to a more homogeneous structure, resulting from the breakdown of the string-like aggregates that exist at higher porosities for high CBD cohesion (see Supporting Information for cross sections of simulated electrode structures with $90 \%$ AM weight loading at $30 \%$ porosity). Some CBD within the porous region still retains its string-like structure, but a majority of the CBD that is compressed within small gaps between AM particles exhibits a more densely-packed mesostructure. However, for higher AM weight loadings (see Supporting Information for cross sections of simulated electrode structures with $96 \%$ AM weight loading at 30\% porosity), this modification of the CBD morphology is not as significant, because the CBD still have enough volume within the pore phase to aggregate into string-like morphology, which is also increasingly oriented orthogonal to the direction of calendering. Therefore, it is apparent that the volume fraction of $\mathrm{CBD}$ within the pore phase is an important mesostructural metric that strongly influences its morphology upon varying its strength of cohesion. For the case of high AM-CBD adhesion and low CBD cohesion, dominant mesostructural characteristics are still maintained upon calendering, which only brings the uniformly CBD-coated AM particles closer together, but does not alter the coating morphology. The effects of calendering on the mesostructure of the electrodes can not be captured in stochastic methods for generating the CBD phase around the experimentallyimaged AM phase.

\subsection{Pore Size Distribution}

The apparent visual differences within the pore space upon changing CBD cohesion and AMCBD adhesion, as shown in Figure 2, can be quantified by measuring the pore size distribution (PSD). Here we report PSD calculated along the medial axis ${ }^{54}$ of the pore phase in the electrodes. For a given PSD $p(r)$, the value of $p(r) \delta r$ indicates the probability of finding a point on the medial axis of the pore phase located at a distance between $r$ and $r+\delta r$ from any solid (i.e., AM or CBD) boundary. As shown in Figure 3(a) for electrodes with $90 \%$ AM weight loading, $50 \%$ porosity, and large CBD cohesion, AM-CBD adhesion does not have a significant effect on the PSD. In addition to PSD, we also compute the mean pore size as a function of the total volume fraction of CBD in the CBD+pore phase. ${ }^{9}$ In Figure 3(c), the mean pore size reduces with increasing CBD fraction, but its value is insensitive to AM-CBD adhesion. When CBD cohesion is small, AMCBD adhesion has a more pronounced effect on the PSD, as seen in Figure 3(b). Upon increasing adhesion, the PSD becomes heaviertailed. This indicates the presence of several large porous regions within the electrode, which can also be visually observed in Figure 2. In addition to the PSD, the mean pore size also increases significantly - up to 2.5 times - when the AM-CBD adhesion is large, especially at low CBD fractions, as shown in Figure 3(d). Controlling adhesion and cohesion during battery processing can remarkably transform the pore space within LIB electrodes, even for the same electrode composition.

At high fractions of the $\mathrm{CBD}$ in the pore phase, the effect of AM-CBD adhesion is significantly reduced even for small CBD cohesion, as shown in Figure 3(d). Thus, there exists a critical CBD volume fraction in the pore phase beyond which the effects of controlling adhesion and cohesion are diminished, as also discussed in the previous section. This critical fraction is strongly dependent on the total AM surface area, and therefore dependent on AM weight loading and AM particle size distribution. This is because once a thin layer of CBD coats the highly adhesive AM surface, the remaining CBD dispersed in the pore phase is screened from the strong adhesive forces and will not preferentially stick on the AM surface.

In addition to PSD, we also calculated the spatial homogeneity of pore sizes within the electrode. In Figures 3(e-f), the local pore size is color coded at various locations within the pore space. When the CBD cohesion is large, 

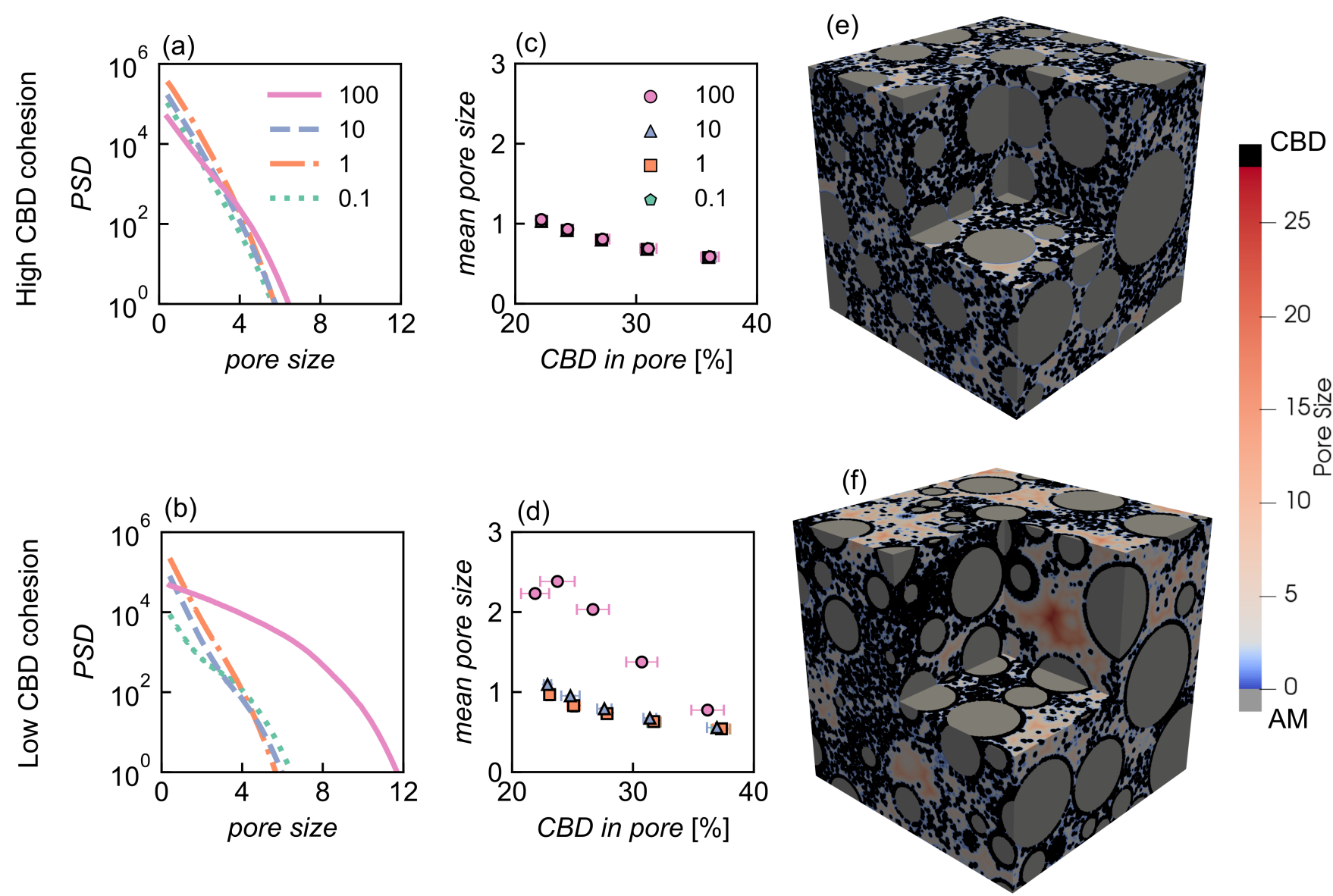

Figure 3: (a-b) Pore size distribution (PSD), (c-d) mean pore size, and (e-f) spatial pore size distribution for high CBD cohesion $(100 k T)$ in $(\mathrm{a}, \mathrm{c}, \mathrm{e})$ and low CBD cohesion $(0.1 k T)$ in $(\mathrm{b}, \mathrm{d}, \mathrm{f})$. PSD in (a,b) correspond to $90 \%$ AM weight loading and $50 \%$ porosity for various AM-CBD adhesion in $k T$ (see legend in (a)). Mean pore size in (c,d) correspond to $90 \%$ AM weight loading averaged over all porosities for various AM-CBD adhesion in $k T$ (see legend in (c)). The horizontal error bars in $(\mathrm{c}, \mathrm{d})$ represent one standard deviation in the data. Spatial pore size distribution in (e) corresponds to low AM-CBD adhesion $(0.1 \mathrm{kT}$ ), and in (f) corresponds to high AM-CBD adhesion $(100 k T)$ for $90 \%$ AM weight loading and 50\% porosity. AM and CBD phases are colored as gray and black respectively, and the pore phase is colored by its local pore size shown in the accompanying color map. The pore size in (a-f) is normalized by the CBD coarse-graining length of $500 \mathrm{~nm}$. 

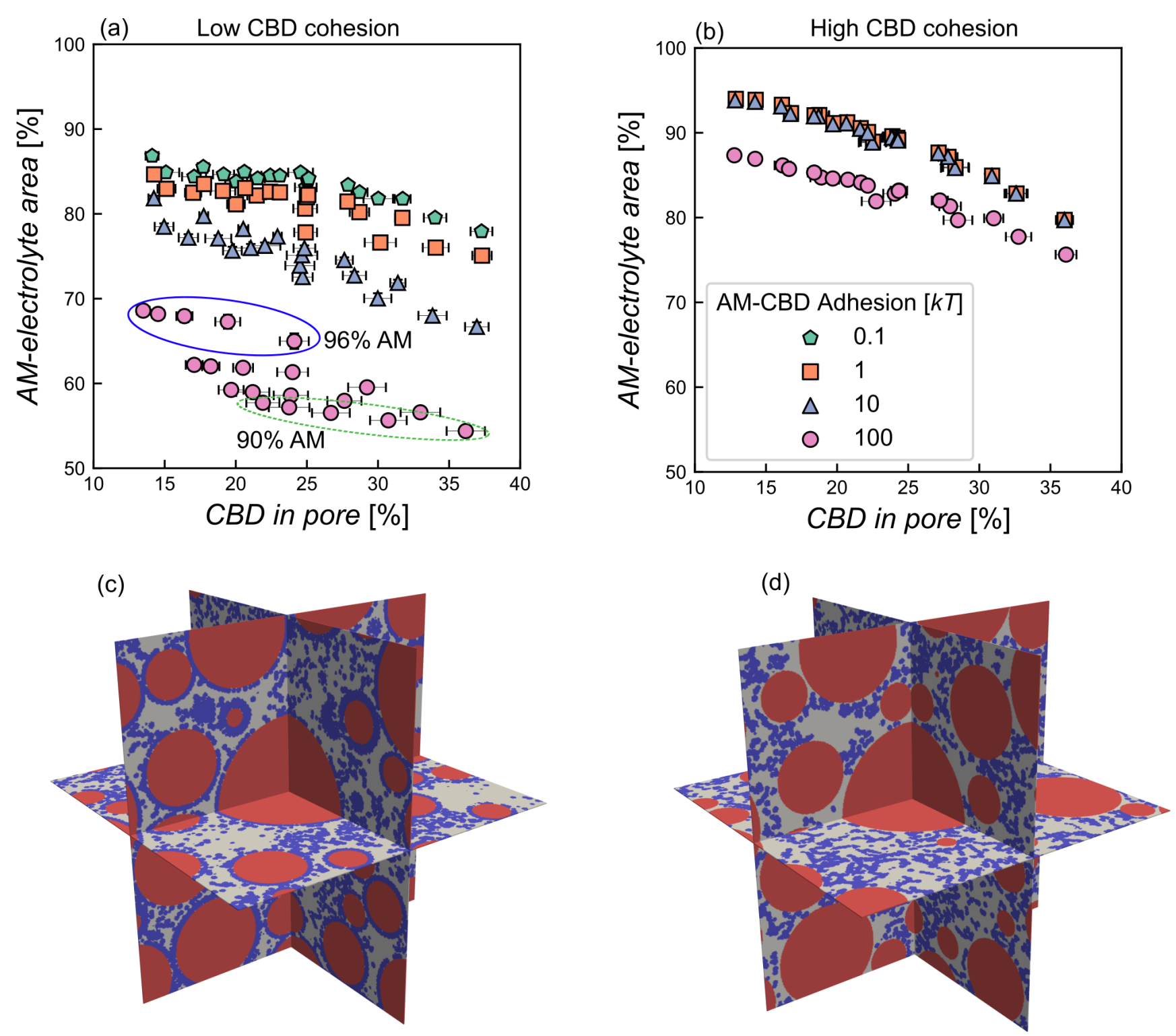

Figure 4: (a-b) AM-electrolyte interface area for various AM-CBD adhesion (see legend in (b)), and (c-d) three orthogonal slices highlighting local CBD and pore space morphology around an AM particle for low CBD cohesion $(0.1 k T)$ in (a,c) and high CBD cohesion $(100 k T)$ in $(\mathrm{b}, \mathrm{d})$. The error bars represent one standard deviation in the data. AM-CBD adhesion is fixed at $100 k T$ in $(\mathrm{c}, \mathrm{d})$. AM, CBD and pore phases are colored as gray, black and white respectively in $(\mathrm{c}, \mathrm{d})$. The solid blue and dashed green ellipses in (a) group data points for all porosities for $96 \%$ and $90 \%$ AM weight loadings respectively. 
the local pore size is homogeneously distributed within the pore space, as shown by the spatial pore size distribution in Figure 3(e). However for large AM-CBD adhesion at small CBD cohesion, the local pore space is highly spatially heterogeneous, as shown in Figure 3(f). This heterogeneity can have significant consequences for ion transport within the electrolyte-filled pores; large spatial heterogeneity of the pore phase would create regions of high ionic concentration rather than uniform ionic distribution, thus resulting in large transport heterogeneity and detrimental effects on battery performance. ${ }^{55,56}$

\subsection{Active Material-Electrolyte Interface Area}

During LIB operation, electrochemical reactions occur at the interfacial area between the electrolyte and the AM surface. These interfaces are primarily present within the mesoscale pore space, but they also exist within the nanoporous CBD that is in contact with the AM surface. Ferraro et al. ${ }^{57}$ suggests that the nanoporous space within the CBD is as accessible for ionic conduction as the mesoscale pore space. The availability of AM-electrolyte interfacial area is crucial to battery functioning and performance. In Figures 4(a-b), the fraction $A$ of AM surface area exposed to electrolytefilled pores is shown as a function of CBD fraction in the $\mathrm{CBD}+$ pore phase. Both the surface area and CBD fraction calculations include $50 \%$ nanoporosity within the CBD, i.e., $50 \%$ of AM and CBD interface area is considered exposed to the electrolyte.

AM-CBD adhesion and CBD cohesion significantly effect the AM-electrolyte interface area. As shown previously, large AM-CBD adhesion causes the CBD material to preferentially diffuse towards and irreversibly stick to the AM particle surface; as a result, the available interfacial area between AM and electrolyte is substantially reduced, as confirmed in Figure 4(a) and also visually seen by the CBD and pore phase morphology in the cross section images in Figure 4(c). This effect is partially ameliorated by the presence of nanoporosity within the
CBD, which still allows some AM-electrolyte contact despite the CBD crowding around AM particles.

CBD cohesion also significantly controls the AM-electrolyte interface area. When the cohesion is high, CBD crowding around an AM particle is substantially suppressed with CBD preferentially forming a network-spanning gel. As a result, larger interfacial AM-electrolyte area is available, as shown in Figure 4(b) and also visually by the CBD phase morphology in Figure 4(d). Furthermore, for low CBD cohesion and high AM-CBD adhesion, the AM weight loading also significantly impacts the AM-electrolyte interface area. When the CBD fraction is high (corresponding to 90\% AM weight loading in Figure 4(a); see dashed green ellipse), AM-electrolyte interface area is less than the case of lower CBD fraction (corresponding to $96 \%$ AM weight loading in Figure 4(a); see solid blue ellipse), because not enough CBD is available to crowd the AM particles. As a result, the available AM-electrolyte area at low CBD cohesion and high AM-CBD adhesion depends both on porosity and AM weight loading. However, when the CBD is highly cohesive, this effect is suppressed and the available AM-electrolyte area depends only on the porosity of the electrode, irrespective of the AM weight loading.

Our predictions of the AM-electrolyte area correspond well with the X-ray and SEM tomography characterization of similar real NMC electrodes at $92 \%$ AM weight loading, which highlighted that $87 \%$ of the AM surface area is contacted by the electrolyte. ${ }^{20}$ More importantly, our simulations highlight that CBD cohesion and AM-CBD adhesion significantly affect the AM-electrolyte area.

\subsection{Ionic Tortuosity}

The significant differences observed in the pore phase topology upon modifying CBD cohesion and AM-CBD adhesion indicate that the effective ionic tortuosity must also vary significantly with these material properties. Ionic tortuosity is estimated from the effective ionic diffusivity in the electrode $D_{\text {eff }}$ through the relation $\tau=$ 

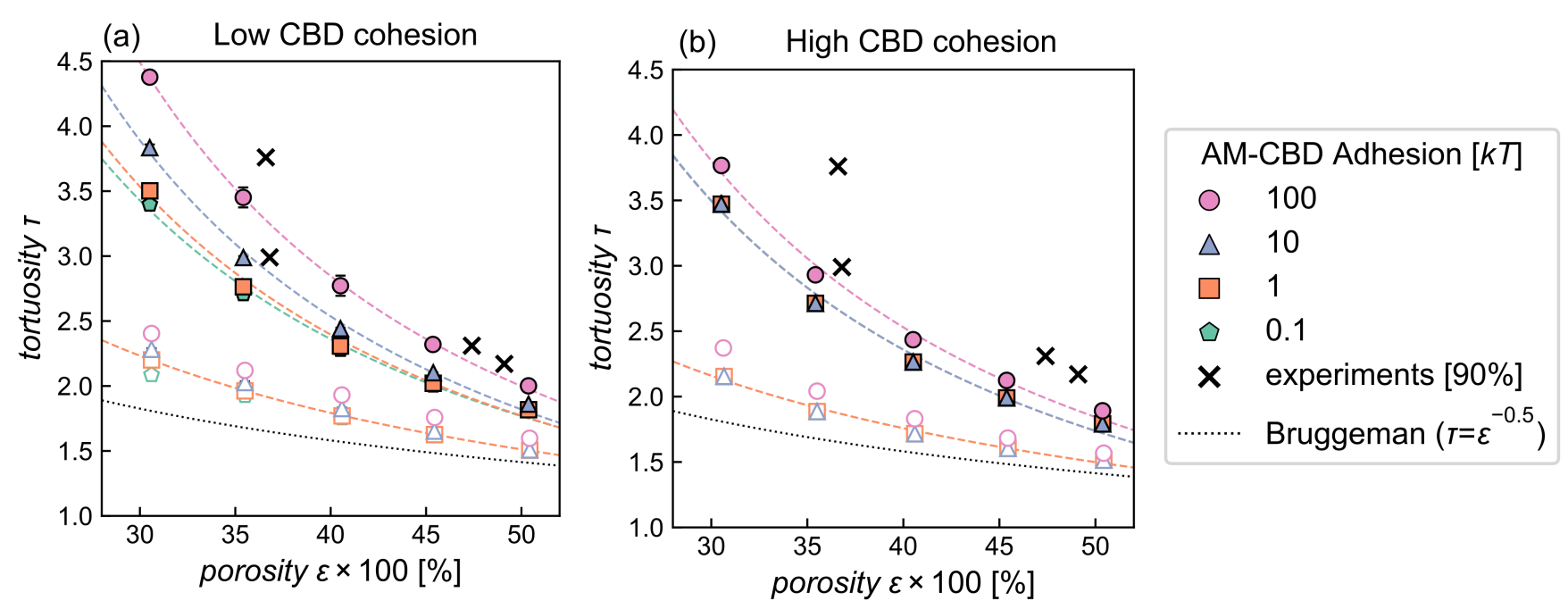

(c) Low AM-CBD adhesion

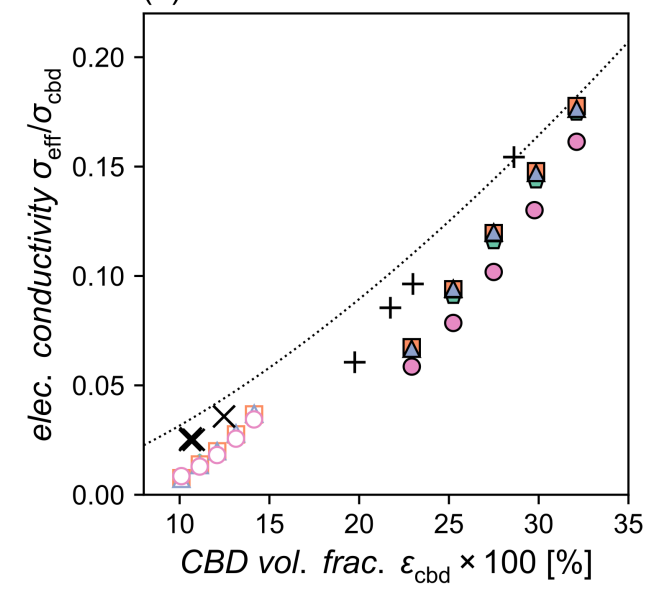

(d) High AM-CBD adhesion

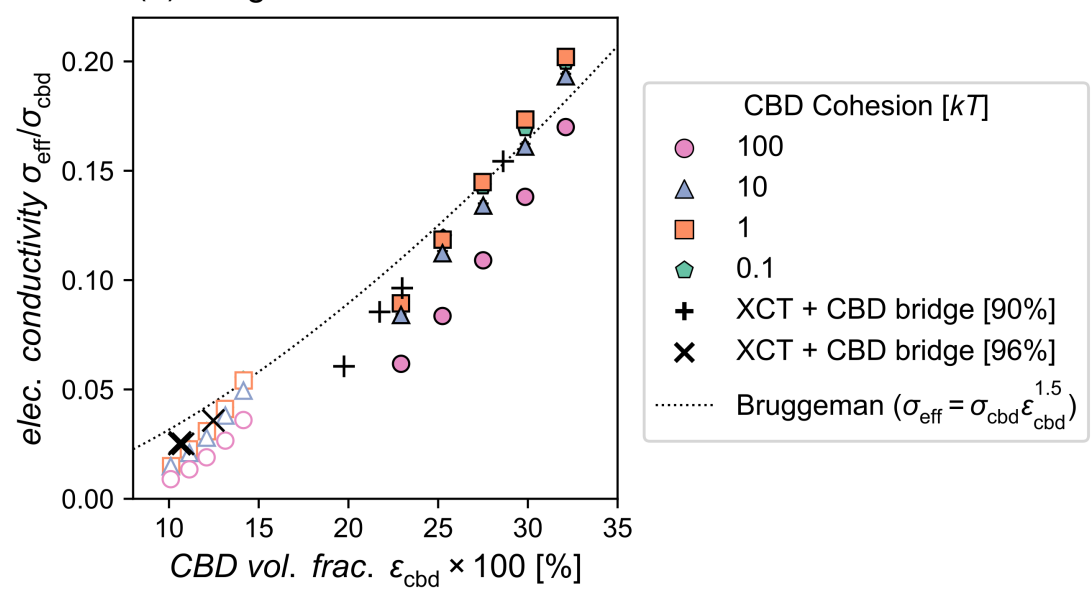

Figure 5: Top row: Ionic tortuosity $\tau$ as a function of porosity $\epsilon$ for (a) low CBD cohesion (0.1 $k T$ ) and (b) high CBD cohesion (100kT) for various AM-CBD adhesion (see legend next to (b)). Closed and open symbols correspond to $90 \%$ and 96\% AM weight loadings respectively. Dashed lines indicate best fit to the equation: $\tau=a \epsilon^{b}$. The dotted line shows the original Bruggeman equation. Black symbols denote data from experiments on NMC electrodes with $90 \%$ AM weight loading (reprinted with permission from ref. ${ }^{38}$ Copyright 2018 IOP Publishing). Bottom row: Effective electronic conductivity $\sigma_{\text {eff }} / \sigma_{\text {cbd }}$ as a function of CBD volume fraction $\epsilon_{\mathrm{cbd}}$ for (c) low AM-CBD adhesion $(0.1 k T)$ and (d) high AM-CBD adhesion $(100 k T)$ for various CBD cohesion (see legend next to (d)). Closed and open symbols correspond to $90 \%$ and $96 \%$ AM weight loadings respectively. Dotted line shows Bruggeman effective medium approximation. Black symbols denote data from XCT images and CBD bridge reconstruction for two AM weight loadings (see legend). 
$D_{0} \epsilon / D_{\text {eff }}$, where $D_{0}$ is the bulk ionic diffusivity in the electrolyte and $\epsilon$ is the total porosity of the electrode, including CBD nanoporosity. The effective ionic diffusivity $D_{\text {eff }}$ is calculated by solving the diffusion equation on electrode mesostructures using the finite element method. Ionic diffusion can occur through CBD as well, with an effective ionic diffusivity that is $5 \%$ of the ionic diffusivity of pure electrolyte. ${ }^{10,24,51}$ Although the importance of ionic conduction through CBD has been recently highlighted in electrochemical modeling of NMC electrodes, ${ }^{57}$ the ion transport is dominated by diffusion through the mesoporous phase and is largely unaffected by diffusion through the CBD.

When the CBD is not cohesive, AM-CBD adhesion significantly affects the ionic tortuosity, with up to $30 \%$ higher ionic tortuosity for the highest AM-CBD adhesion compared to lowest adhesion at 30\% electrode porosity, as shown in Figure 5(a). Although the electrodes with the largest AM-CBD adhesion (and low CBD cohesion) exhibit large pore sizes, as described in Section 4.2, the ionic tortuosity is counterintuitively higher than the electrodes with small adhesion for the same porosity. This counterintuitive effect occurs because the spatial arrangement of $\mathrm{AM}$ particles in mesostructures with large AM-CBD adhesion and small CBD cohesion is highly heterogeneous (see Figure 2), resulting in co-existing regions of close-packed CBD-coated AM particles and regions of high porosity. The regions of close-packed AM particles is highly constrictive to the diffusion of ions, thus resulting in increased ionic tortuosity. Upon increasing the AM weight loading, the ionic tortuosity is significantly reduced at all porosities as shown in Figures 5(a) and (b), although the effect of AM-CBD adhesion still persists.

For a highly cohesive CBD phase, the effect of AM-CBD adhesion is not so pronounced, as shown in Figure 5(b). Furthermore, the ionic tortuosity is lower in the case of highly cohesive CBD when the AM-CBD adhesion is high. As described previously, high cohesion in the CBD phase restricts the dramatic effects of AM-CBD adhesion on the mesostructure, thus resulting in a more uniform spatial distribution of the
AM particles that do not contain close-packed regions of high ionic constriction. When the AM-CBD adhesion is low, CBD cohesion does not affect the ionic tortuosity significantly.

Our predictions of ionic tortuosity correspond well with experiments on a similar electrode system containing 90\% AM weight loading of NMC532 AM particles, ${ }^{38}$ as shown by black symbols in Figures 5(a) and (b). Although a one-to-one correspondence between experimental data and numerical predictions is always challenging from a lack of the knowledge of spatial material topology within experimental electrodes, our numerical results importantly highlight that a careful control of AM-CBD adhesion and CBD cohesion can significantly modify ionic tortuosity by up to $\sim 30 \%$.

The numerical predictions of ionic tortuosity was also compared to the classical Bruggeman effective medium equation, $\tau=\epsilon^{0.5}$, which has historically been used to predict ionic transport in tortuous battery electrodes. ${ }^{58}$ We find that the Bruggeman equation significantly underpredicts ionic tortuosity, which is consistent with prior experimental ${ }^{38}$ and numerical results, ${ }^{24,59}$ and the deviations from Bruggeman equation are more pronounced for lower AM weight loadings, as shown in Figures 5(a) and (b). However, all the data is well-fitted by a generalized equation for effective ionic tortuosity, $\tau=a \epsilon^{b}$, where $a$ and $b$ are the fitting parameters. ${ }^{38}$

\subsection{Effective Electronic Conduc- tivity}

The AM phase in the cathode of LIB has poor electronic conductivity, and electronic conduction primarily occurs through the carboncontaining CBD phase. As a result the CBD phase morphology has a significant effect on the effective electronic conductivity $\sigma_{\text {eff }}$ of the LIB cathodes. The effective electronic conductivity is calculated by solving the electronic current conservation equation, where the CBD phase conductivity is set to $\sigma_{\text {cbd }}=15.9 \mathrm{~S} \mathrm{~m}^{-} 1$, AM phase conductivity is set to $0.18 \mathrm{~S} \mathrm{~m}^{-1}$, and the electrolyte is non-conducting. ${ }^{50,60}$

As the volume fraction of the CBD phase is in- 
creased, the electronic conductivity expectedly increases, as shown by the variation of ratio $\sigma_{\text {eff }} / \sigma_{\text {cbd }}$, with the CBD volume fraction $\epsilon_{\text {cbd }}$ in Figures 5(c) and (d). When the AM-CBD adhesion is low, the effective electronic conductivity decreases moderately with increasing CBD cohesion, as shown in Figure 5(c). The highly cohesive CBD phase aggregates to form a gellike network, which is preferentially oriented orthogonal to the direction of drying and calendering. As a result, the out-of-plane conductivity measured here decreases for the highly cohesive CBD, and this effect is magnified at lower porosities, i.e., higher $\epsilon_{\mathrm{cbd}}$. When the AM$\mathrm{CBD}$ adhesion is high, the low CBD cohesion electrode is $\sim 20 \%$ more conducting than the high CBD cohesion electrode, as shown in Figure 5(d). This happens because low CBD cohesion results in long-ranged clusters of closedpacked CBD-coated AM particles, thereby providing several potentially percolating pathways for electronic conduction.

The variation of $\sigma_{\text {eff }}$ with $\mathrm{CBD}$ cohesion is much less significant at higher AM weight loadings, as shown in Figures 5(c) and (d). The volume of available CBD in these electrodes is low, and the CBD phase is unable to form longranged gel-like mesostructures, as described in Section 2. Consequently, the morphological differences between low and high cohesion CBD are small, which reflects in their effective electronic conductivities.

The predictions of effective electronic conductivity correspond well with data obtained from numerically generated electrodes from XCT images of AM particles ${ }^{18}$ and bridge-based reconstruction of the CBD phase, ${ }^{24}$ for both high and low AM weight loading, as shown by black symbols in Figures 5(c) and (d). Particularly, the data corresponds well with electrodes possessing high AM-CBD adhesion and low CBD cohesion resulting in a long-ranged connected CBD phase, which is consistent with the CBD morphology obtained from bridge-based CBD reconstruction rules. ${ }^{24}$

Unlike ionic tortuosity of the electrodes, the effective electronic conductivity corresponds well with predictions from Bruggeman effective medium theory: ${ }^{61,62} \sigma_{\text {eff }}=\sigma_{\text {cbd }} \epsilon_{\text {cbd }}^{1.5}$, as shown by the dotted lines in Figures 5(c) and (d). Traditionally, the Bruggeman effective theory has been shown to incorrectly predict the effective electronic conductivity of the electrodes, ${ }^{62-64}$ following the commonly adopted assumption that electronic conduction occurs equivalently through the entire solid (AM and CBD) phase. The present results demonstrate that if the relative contributions of $\mathrm{AM}$ and $\mathrm{CBD}$ phases towards electronic conduction are taken into account, Bruggeman effective medium theory offers reasonable predictions of the effective electronic conductivity. Conversely, effective electronic conductivity - which is often easy to measure in real electrodes ${ }^{63}$ - can provide reasonably accurate estimates of the CBD volume fraction that is quite challenging to measure directly in experiments.
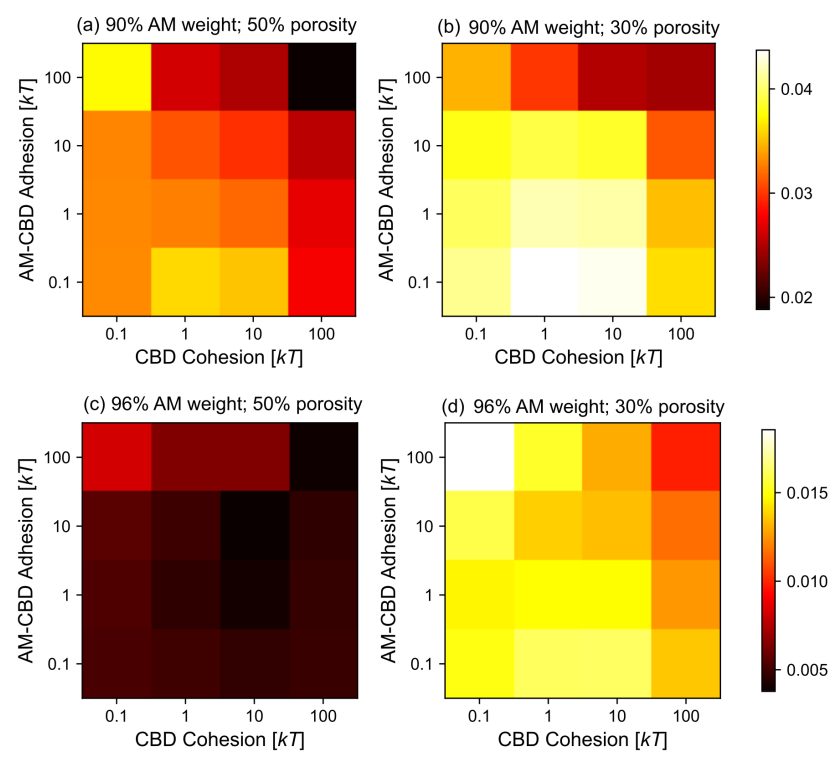

Figure 6: Variation of electrode figure of merit $M$ with AM-CBD adhesion and CBD cohesion for electrodes for two AM weight loadings and two porosities (see top of each panel). The top and bottom color maps denote the range of $M$ in $(\mathrm{a}, \mathrm{b})$ and $(\mathrm{c}, \mathrm{d})$ respectively.

\subsection{Electrode Performance}

The performance of a battery electrode during operation (charging and discharging) is primarily governed simultaneously by the efficiency of ionic diffusion, the efficiency of electronic conduction, and the available area of AM particles 
to the electrolyte for electrochemical reactions. These transport phenomena were characterized in the previous sections by ionic tortuosity $\tau$, effective electronic conductivity $\sigma_{\text {eff }}$, and the available AM-electrolyte area $A$ respectively.

We describe the combined transport performance of a battery electrode using a figure of merit $M$ defined as $M=\left(\frac{A}{\tau}\right)\left(\frac{\sigma_{\text {eff }}}{\sigma_{\text {cbd }}}\right)$. This dimensionless number provides a first-order description of electrochemical transport efficiency within battery electrodes. Note that like $A$ and $\tau$, the ratio $\left(\frac{\sigma_{\text {eff }}}{\sigma_{\text {cbd }}}\right)$ is a purely mesostructural quantity and does not depend on the intrinsic CBD electronic conductivity. Consequently, the parameter $M$ estimates transport efficiency entirely as a result of electrode mesostructure rather than intrinsic material properties. The parameter $M$ was calculated from the results of the previous sections for electrodes at various AM weight loadings, electrode porosities, CBD cohesions and AM-CBD adhesions. Figure 6 displays the variation of $M$ with AM-CBD adhesion and CBD cohesion for two AM weight loadings and two porosities.

The variation of $M$ with AM-CBD cohesion and CBD adhesion is highly non-trivial. For 90\% AM weight loading and 50\% electrode porosity, the most transport-efficient electrodes are equivalently found when the AM-CBD adhesion is low and $\mathrm{CBD}$ cohesion is moderate, and when CBD cohesion is low and AM-CBD adhesion is high, as shown in Fig 6(a). For the former case, transport efficiency is maximized through a combination of low ionic tortuosity and large AM-electrolyte area, whereas for the latter case, transport efficiency is maximized entirely through increased electronic conductivity. The trends in $M$ remain consistent upon calendering the electrodes to $30 \%$ porosity, even though the electrodes become more transport efficient through increased electronic conductivity within the dense CBD phase, as shown in Fig 6(b). Furthermore, the variation of $M$ with AM-CBD adhesion and CBD cohesion is similar for electrodes with $96 \% \mathrm{AM}$ weight loading (see Figs 6(c) and (d)), even though the electrodes on average are only one-third as efficient as electrodes with $90 \%$ AM weight loading, resulting from significantly lower electronic conductivity.
The case of high CBD cohesion and high AMCBD adhesion is consistently the least efficient in transport, resulting from simultaneous high ionic tortuosity, low electronic conductivity and low AM-electrolyte interface area.

These results highlight the intricate interdependence of transport phenomena in LIB electrodes with AM-CBD adhesion and CBD cohesion. Additionally, these results also demonstrate that through a careful control of adhesion in LIB electrode components, it is possible to achieve significant transport enhancement. Although the figure of merit introduced in this study provides a simple surrogate for electrochemical efficiency, a detailed electrochemical model such as described in Ferraro et al., ${ }^{57}$ along with advanced simulation methods for characterizing ionic diffusion, ${ }^{65,66}$ will provide further insights into the role of AM-CBD adhesion and CBD cohesion on battery performance. Such analyses constitute an important part of our future work.

\section{Conclusion and Outlook}

We have presented a comprehensive computational study that describes a promising strategy to engineer LIB electrode mesostructures for enhanced electrochemical transport: controlling the CBD phase cohesion and adhesion between AM particles and CBD phase. Importantly, this strategy is compatible with traditional electrode manufacturing methods. Using a combination of granular and colloidal dynamics simulations involving thousands of AM particles and millions of CBD coarse-grained particles for several AM weight loadings and electrode porosities, we have shown the dominant effect of these material parameters on the mesostructure and transport in LIB electrodes and how they can be carefully controlled to maximize electrode performance for a given composition of the electrode. The simulations presented in this study are large in scale, effectively representing the electrode at experimentally-relevant length scales of $100 \mu \mathrm{m}$. Furthermore, these simulations examine the effects of calendering on the mesostructure of the 
electrodes, which is not possible in stochastic computational methods for simulating the CBD phase.

The cohesion of the CBD phase and adhesion between AM-CBD phases significantly control the CBD and pore phase morphology. Large CBD cohesion causes the CBD to form disconnected string-like networks, instead of a homogeneous distribution. Large AM-CBD adhesion causes CBD particles to diffuse towards and stick to AM particles, resulting in significantly larger pore size distributions, and significantly lower AM-electrolyte interface area. However, large CBD cohesion was shown to counteract some of these effects, by providing a dynamical competition between CBD clustering and AMCBD coating.

The analysis of transport-relevant parameters such as ionic tortuosity, electronic conductivity and AM-electrolyte interface area reveal that cohesion and adhesion can significantly modulate electrochemical transport. High AM-CBD adhesion results in reduced AM-electrolyte area and increased ionic tortuosity, while simultaneously also increasing the electronic conductivity. CBD cohesion has a less dominating effect on various transport properties, but can counter-balance some of the effects induced by large AM-CBD adhesion. AM weight loading was also shown to play a crucial role: the effect of cohesion and adhesion is significantly minimized for higher AM weight loadings. To put all the transport analysis into perspective, we formulated a figure of merit describing the complete electrochemical performance of an electrode to the first order. We showed that through the controlling of cohesion and adhesion, electrode performance can be doubled, thus providing opportunities for material property optimization.

This work could open up avenues for rational electrode design based on simulations that incorporate realistic material properties and also provide predictions of material properties towards optimizing electrochemical transport. Because the simulations are physics-based, they can be extended to optimize other important properties of LIB electrodes relevant to their production or operation, such as its mechanical strength and the rheology (or manufacturability) of its precursor slurry. These constitute an important part of our future work.

\section{Supporting Information Avail- able}

Electrode dynamics simulations; transport calculations; cross sections of simulated electrode structures for intermediate AM:CBD weight loadings at $30 \%$ and $50 \%$ porosities (Figures S1S5); mesostructural and transport properties of simulated electrodes for intermediate AM:CBD weight loadings (Table S1)

Acknowledgement The authors acknowledge Bradley Trembacki for helpful discussions, and Christine C. Roberts for valuable feedback on the manuscript. This work was funded by the U.S. Department of Energy (DOE) Vehicle Technologies Office CAEBAT III program led by technology manager Brian Cunningham. Final manuscript preparation was also sponsored by the Laboratory Directed Research and Development program at Sandia National Laboratories. This work was performed in part at the Center for Integrated Nanotechnologies, a U.S. Department of Energy and Office of Basic Energy Sciences user facility. Sandia National Laboratories is a multimission laboratory managed and operated by National Technology and Engineering Solutions of Sandia, LLC, a wholly owned subsidiary of Honeywell International, Inc., for the U.S. Department of Energy's National Nuclear Security Administration under Contract No. DE-NA-0003525. This paper describes objective technical results and analysis. Any subjective views or opinions that might be expressed in the paper do not necessarily represent the views of the U.S. Department of Energy or the United States Government.

\section{References}

(1) Tarascon, J.; Armand, M. Materials for Sustainable Energy: A Collection of PeerReviewed Research and Review Articles 
from Nature Publishing Group; 2011; pp 171-179.

(2) Cabana, J.; Monconduit, L.; Larcher, D.; Palacin, M. R. Beyond Intercalation-based Li-ion Batteries: The State of the Art and Challenges of Electrode Materials Reacting through Conversion Reactions. Adv. Mater. 2010, 22, E170-E192.

(3) Lai, W.; Erdonmez, C. K.; Marinis, T. F.; Bjune, C. K.; Dudney, N. J.; Xu, F.; Wartena, R.; Chiang, Y.-M. UltrahighEnergy-Density Microbatteries Enabled by New Electrode Architecture and Micropackaging Design. Adv. Mater. 2010, 22, E139-E144.

(4) Bae, C.-J.; Erdonmez, C. K.; Halloran, J. W.; Chiang, Y.-M. Design of Battery Electrodes with Dual-Scale Porosity to Minimize Tortuosity and Maximize Performance. Adv. Mater. 2013, 25, 12541258.

(5) Wu, H.; Yu, G.; Pan, L.; Liu, N.; McDowell, M. T.; Bao, Z.; Cui, Y. Stable Li-ion Battery Anodes by in-situ Polymerization of Conducting Hydrogel to Conformally Coat Silicon Nanoparticles. Nat. Commun. 2013, 4, 1943.

(6) Li, L.; Erb, R. M.; Wang, J.; Wang, J.; Chiang, Y.-M. Fabrication of LowTortuosity Ultrahigh-Area-Capacity Battery Electrodes through Magnetic Alignment of Emulsion-Based Slurries. Adv. Energy Mater. 2019, 9, 1802472.

(7) Gao, H.; Wu, Q.; Hu, Y.; Zheng, J.; Amine, K.; Chen, Z. Revealing the RateLimiting Li-Ion Diffusion Pathway in Ultrathick Electrodes for Li-Ion Batteries. $J$. Phys. Chem. Lett. 2018, 9, 5100-5104.

(8) Ma, F.; Fu, Y.; Battaglia, V.; Prasher, R. Microrheological Modeling of Lithium Ion Battery Anode Slurry. J. Power Sources 2019, 438, 226994.

(9) Zielke, L.; Hutzenlaub, T.; Wheeler, D. R.; Manke, I.; Arlt, T.; Paust, N.;
Zengerle, R.; Thiele, S. A Combination of X-Ray Tomography and Carbon Binder Modeling: Reconstructing the Three Phases of LiCoO2Li-Ion Battery Cathodes. Adv. Energy Mater. 2014, 4, 1301617.

(10) Zielke, L.; Hutzenlaub, T.; Wheeler, D. R.; Chao, C.-W.; Manke, I.; Hilger, A.; Paust, N.; Zengerle, R.; Thiele, S. ThreePhase Multiscale Modeling of a $\mathrm{LiCoO} 2$ Cathode: Combining the Advantages of FIB-SEM Imaging and X-Ray Tomography. Adv. Energy Mater. 2015, 5, 1401612.

(11) Morelly, S. L.; Gelb, J.; Iacoviello, F.; Shearing, P. R.; Harris, S. J.; Alvarez, N. J.; Tang, M. H. ThreeDimensional Visualization of Conductive Domains in Battery Electrodes with Contrast-Enhancing Nanoparticles. ACS Appl. Energy Mater. 2018, 1, 4479-4484.

(12) Kraytsberg, A.; Ein-Eli, Y. Conveying Advanced Li-ion Battery Materials into Practice The Impact of Electrode Slurry Preparation Skills. Adv. Energy Mater. 2016, 6, 1600655 .

(13) Lu, P. J.; Zaccarelli, E.; Ciulla, F.; Schofield, A. B.; Sciortino, F.; Weitz, D. A. Gelation of Particles with Short-range Attraction. Nature 2008, 453, 499.

(14) Smay, J. E.; Gratson, G. M.; Shepherd, R. F.; Cesarano III, J.; Lewis, J. A. Directed Colloidal Assembly of 3D Periodic Structures. Adv. Mater. 2002, 14, 1279-1283.

(15) Persson, B. N.; Scaraggi, M. Theory of Adhesion: Role of Surface Roughness. J. Chem. Phys. 2014, 141, 124701.

(16) Lewis, J. A. Colloidal Processing of Ceramics. J. Am. Ceram. Soc. 2000, 83, 2341-2359. 
(17) Chen, H.; Ling, M.; Hencz, L.; Ling, H. Y.; Li, G.; Lin, Z.; Liu, G.; Zhang, S. Exploring Chemical, Mechanical, and Electrical Functionalities of Binders for Advanced Energy-Storage Devices. Chem. Rev. 2018, 118, 8936 - 8982 .

(18) Ebner, M.; Geldmacher, F.; Marone, F.; Stampanoni, M.; Wood, V. X-Ray Tomography of Porous, Transition Metal Oxide Based Lithium Ion Battery Electrodes. Adv. Energy Mater. 2013, 3, 845-850.

(19) Moroni, R.; Börner, M.; Zielke, L.; Schroeder, M.; Nowak, S.; Winter, M.; Manke, I.; Zengerle, R.; Thiele, S. MultiScale Correlative Tomography of a LiIon Battery Composite Cathode. Sci. Rep. 2016, 6, 30109.

(20) Besnard, N.; Etiemble, A.; Douillard, T.; Dubrunfaut, O.; Tran-Van, P.; Gautier, L.; Franger, S.; Badot, J.-C.; Maire, E.; Lestriez, B. Multiscale Morphological and Electrical Characterization of Charge Transport Limitations to the Power Performance of Positive Electrode Blends for Lithium-Ion Batteries. $A d v$. Energy Mater. 2017, 7, 1602239.

(21) Rajendra, T.; Mistry, A. N.; Patel, P.; Ausderau, L.; Xiao, X.; Mukherjee, P. P.; Nelson, G. Quantifying Transport, Geometrical and Morphological Parameters in Li-ion Cathode Phases using X-ray Microtomography. ACS Appl. Mater. Interfaces 2019, 11, 19933-19942.

(22) Daemi, S. R.; Tan, C.; Volkenandt, T.; Cooper, S. J.; Palacios-Padros, A.; Cookson, J.; Brett, D. J. L.; Shearing, P. R. Visualizing the Carbon Binder Phase of Battery Electrodes in Three Dimensions. ACS Appl. Energy Mater. 2018, 1, 37023710 .

(23) Mistry, A.; Smith, K.; Mukherjee, P. Secondary-Phase Stochastics in LithiumIon Battery Electrodes. ACS Appl. Mater. Interfaces 2018, 10, 6317-6326.
(24) Trembacki, B. L.; Mistry, A. N.; Noble, D. R.; Ferraro, M. E.; Mukherjee, P. P.; Roberts, S. A. Mesoscale Analysis of Conductive Binder Domain Morphology in Lithium-Ion Battery Electrodes. J. Electrochem. Soc. 2018, 165, E725-E736.

(25) Westhoff, D.; Manke, I.; Schmidt, V. Generation of Virtual Lithium-ion Battery Electrode Microstructures based on Spatial Stochastic Modeling. Comput. Mater. Sci. 2018, 151, 53-64.

(26) Trembacki, B. L.; Noble, D. R.; Ferraro, M. E.; Roberts, S. A. Mesoscale Effects of Composition and Calendering in Lithium-Ion Battery Composite Electrodes. J. Electrochem. Energy Convers. Storage 2020, 17, 041001.

(27) Forouzan, M. M.; Chao, C.-W.; Bustamante, D.; Mazzeo, B. A.; Wheeler, D. R. Experiment and Simulation of the Fabrication Process of Lithium-ion Battery Cathodes for Determining Microstructure and Mechanical Properties. J. Power Sources 2016, 312, 172-183.

(28) Stershic, A.; Simunovic, S.; Nanda, J. Modeling the Evolution of Lithium-ion Particle Contact Distributions using a Fabric Tensor Approach. J. Power Sources 2015, 297, 540-550.

(29) Ludwig, B.; Liu, J.; Liu, Y.; Zheng, Z.; Wang, Y.; Pan, H. Simulation of Micro/Nanopowder Mixing Characteristics for Dry Spray Additive Manufacturing of Li-ion Battery Electrodes. J. Micro NanoManufacturing 2017, 5, 040902.

(30) Cerbelaud, M.; Lestriez, B.; Videcoq, A.; Ferrando, R.; Guyomard, D. Understanding the Structure of Electrodes in Li-Ion Batteries: A Numerical Study. J. Electrochem. Soc. 2015, 162, A1485-A1492.

(31) Ngandjong, A.; Rucci, A.; Maiza, M.; Shukla, G.; Vazquez-Arenas, J.; Franco, A. Multiscale Simulation Platform 
Linking Lithium Ion Battery Electrode Fabrication Process with Performance at the Cell Level. J. Phys. Chem. Lett. 2017, 8, 5966-5972.

(32) Zhu, M.; Park, J.; Sastry, A. M. Particle Interaction and Aggregation in Cathode Material of Li-Ion Batteries: A Numerical Study. J. Electrochem. Soc. 2011, 158, A1155.

(33) Rucci, A.; Ngandjong, A. C.; Primo, E. N.; Maiza, M.; Franco, A. A. Tracking Variabilities in the Simulation of Lithium Ion Battery Electrode Fabrication and Its Impact on Electrochemical Performance. Electrochim. Acta 2019, 312, 168-178.

(34) Chouchane, M.; Rucci, A.; Lombardo, T.; Ngandjong, A. C.; Franco, A. A. Lithium Ion Battery Electrodes Predicted From Manufacturing Simulations: Assessing the Impact of the Carbon-Binder Spatial Location on the Electrochemical Performance. J. Power Sources 2019, 444, 227285 .

(35) Lombardo, T.; Hoock, J. B.; Primo, E. N.; Ngandjong, A. C.; Duquesnoy, M.; Franco, A. A. Accelerated Optimization Methods for Force-field Parametrization in Battery Electrode Manufacturing Modeling. Batteries Supercaps 2020, 3, 1-11.

(36) Franco, A.; Rucci, A.; Brandell, D.; Frayret, C.; Gaberscek, M.; Jankowski, P.; Johansson, P. Boosting Rechargeable Batteries R\&D by Multiscale Modeling: Myth or Reality. Chem. Rev. 2019, 119, 45694627.

(37) Ryan, E. M.; Mukherjee, P. P. Mesoscale Modeling in Electrochemical Devices - a Critical Perspective. Prog. Energy Combust. Sci. 2019, 71, 118-142.

(38) Usseglio-Viretta, F. L.; Colclasure, A.; Mistry, A. N.; Claver, K. P. Y.; Pouraghajan, F.; Finegan, D. P.; Heenan, T. M.; Abraham, D.; Mukherjee, P. P.;
Wheeler, D. Resolving the Discrepancy in Tortuosity Factor Estimation for Li-ion Battery Electrodes through Micro-Macro Modeling and Experiment. J. Electrochem. Soc. 2018, 165, A3403-A3426.

(39) De Graaf, J.; Poon, W. C.; Haughey, M. J.; Hermes, M. Hydrodynamics Strongly affect the Dynamics of Colloidal Gelation but not Gel Structure. Soft Matter 2019, 15, 10-16.

(40) Brilliantov, N. V.; Spahn, F.; Hertzsch, J.M.; Poschel, T. Model for Collisions in Granular Gases. Phys. Rev. E 1996, 53, 5382 .

(41) Barthel, E. Adhesive Elastic Contacts: JKR and More. J. Phys. D: Appl. Phys. 2008, 41, 163001.

(42) Plimpton, S. Fast Parallel Algorithms for Short-Range Molecular Dynamics. $J$. Comp. Phys. 1995, 117, 1-19.

(43) Vasconcelos, L. S. D.; Xu, R.; Li, J.; Zhao, K. Grid Indentation Analysis of Mechanical Properties of Composite Electrodes in Li-ion Batteries. Extreme Mech. Lett. 2016, 9, 495-502.

(44) Jaiser, S.; Kumberg, J.; Klaver, J.; Urai, J. L.; Schabel, W.; Schmatz, J.; Scharfer, P. Microstructure Formation of Lithium-ion Battery Electrodes during Drying - An ex-situ Study using Cryogenic Broad Ion Beam Slope-cutting and Scanning Electron Microscopy (Cryo-BIBSEM). J. Power Sources 2017, 345, 97107.

(45) Font, F.; Protas, B.; Richardson, G.; Foster, J. M. Binder Migration during Drying of Lithium-ion Battery Electrodes: Modelling and Comparison to Experiment. $J$. Power Sources 2018, 393, 177-185.

(46) Yoo, M.; Frank, C. W.; Mori, S. Interaction of Poly(vinylidene Fluoride) with Graphite Particles. 1. Surface Morphology of a Composite Film and its Relation 
to Processing Parameters. Chem. Mater. 2003, 15, 850-861.

(47) Sangrós Giménez, C.; Finke, B.; Schilde, C.; Froböse, L.; Kwade, A. Numerical Simulation of the Behavior of Lithium-ion Battery Electrodes during the Calendaring Process via the Discrete Element Method. Powder Tech. 2019, $349,1-11$.

(48) Sangrós Giménez, C.; Schilde, C.; Froböse, L.; Ivanov, S.; Kwade, A. Mechanical, Electrical, and Ionic Behavior of Lithium-ion Battery Electrodes via Discrete Element Method Simulations. Energy Technol. 2019, 8, 1900180.

(49) Park, Y.-S.; Oh, E.-S.; Lee, S.-M. Effect of Polymeric Binder type on the Thermal Stability and Tolerance to Roll-Pressing of Spherical Natural Graphite Anodes for Liion Batteries. J. of Power Sources 2014, 248, 1191-1196.

(50) Grillet, A. M.; Humplik, T.; Stirrup, E. K.; Roberts, S. A.; Barringer, D. A.; Snyder, C. M.; Janvrin, M. R.; Apblett, C. A. Conductivity Degradation of Polyvinylidene Fluoride Composite Binder during Cycling: Measurements and Simulations for Lithiumion Batteries. J. Electrochem. Soc. 2016, 163, A1859-A1871.

(51) Stephenson, D. E.; Walker, B. C.; Skelton, C. B.; Gorzkowski, E. P.; Rowenhorst, D. J.; Wheeler, D. R. Modeling 3d Microstructure and Ion Transport in Porous Li-ion Battery Electrodes. J. Electrochem. Soc. 2011, 158, A781.

(52) Roberts, S. A.; Mendoza, H.; Brunini, V. E.; Noble, D. R. A Verified Conformal Decomposition Finite Element Method for Implicit, ManyMaterial Geometries. J. Comp. Phys. 2018, 375, 352-367.

(53) Trappe, V.; Prasad, V.; Cipelletti, L.; Segre, P. N.; Weitz, D. A. Jamming Phase
Diagram for Attractive Particles. Nature 2001, 411, 772-775.

(54) Lindquist, W. B.; Lee, S.-M.; Coker, D. A.; Jones, K. W.; Spanne, P. Medial Axis Analysis of Void Structure in Three-Dimensional Tomographic Images of Porous Media. J. Geophys. Res.: Solid Earth 1996, 101, 8297-8310.

(55) Kehrwald, D.; Shearing, P. R.; Brandon, N. P.; Sinha, P. K.; Harris, S. J. Local Tortuosity Inhomogeneities in a Lithium Battery Composite Electrode. J. Electrochem. Soc. 2011, 158, A1393.

(56) Harris, S. J.; Lu, P. Effects of Inhomogeneities - Nanoscale to Mesoscale on the Durability of LiIon Batteries. J. Phys. Chem. C 2013, 117, 6481-6492.

(57) Ferraro, M. E.; Trembacki, B. L.; Brunini, V. E.; Noble, D. R.; Roberts, S. A. Electrode Mesoscale as a Collection of Particles: Coupled Electrochemical and Mechanical Analysis of NMC Cathodes. J. Electrochem. Soc. 2020, 16\%, 013543.

(58) Tjaden, B.; Cooper, S. J.; Brett, D. J.; Kramer, D.; Shearing, P. R. On the Origin and Application of the Bruggeman Correlation for Analysing Transport Phenomena in Electrochemical Systems. Curr. Opin. Chem. Eng. 2016, 12, 44-51.

(59) Chung, D.-W.; Ebner, M.; Ely, D. R.; Wood, V.; Edwin García, R. Validity of the Bruggeman Relation for Porous Electrodes. Modell. Simul. Mater. Sci. Eng. 2013, 21, 074009.

(60) Trembacki, B. L.; Noble, D. R.; Brunini, V. E.; Ferraro, M. E.; Roberts, S. A. Mesoscale Effective Property Simulations Incorporating Conductive Binder. J. Electrochem. Soc. 2017, 164, E3613-E3626.

(61) Srivastava, I.; Sadasivam, S.; Smith, K. C.; Fisher, T. S. Combined 
Microstructure and Heat Conduction Modeling of Heterogeneous Interfaces and Materials. J. Heat Transfer 2013, 135, 061603.

(62) Vadakkepatt, A.; Trembacki, B.; Mathur, S. R.; Murthy, J. Y. Bruggeman's Exponents for Effective Thermal Conductivity of Lithium-ion Battery Electrodes. J. Electrochem. Soc. 2016, 163, A119-A130.

(63) Peterson, S. W.; Wheeler, D. R. Direct measurements of effective electronic transport in porous li-ion electrodes. J. Electrochem. Soc. 2014, 161, A2175-A2181.

(64) Nevers, D. R.; Peterson, S. W.; Robertson, L.; Chubbuck, C.; Flygare, J.; Cole, K.; Wheeler, D. R. The Effect of Carbon Additives on the Microstructure and Conductivity of Alkaline Battery Cathodes. J. Electrochem. Soc. 2014, 161, A1691-A1697.

(65) Chouchane, M.; Primo, E. N.; Franco, A. A. Mesoscale Effects in the Extraction of the Solid-State Lithium Diffusion Coefficient Values of Battery Active Materials: Physical Insights From 3d Modeling. J. Phys. Chem. Lett. 2020, 11, 2775-2780.

(66) Shodiev, A.; Primo, E. N.; Chouchane, M.; Lombardo, T.; Ngandjong, A. C.; Rucci, A.; Franco, A. A. 4d-Resolved Physical Model for Electrochemical Impedance Spectroscopy of $\mathrm{Li}$ (Ni1-x-yMnxCoy) O2-Based Cathodes in Symmetric Cells: Consequences in Tortuosity Calculations. J. Power Sources 2020, 454, 227871. 


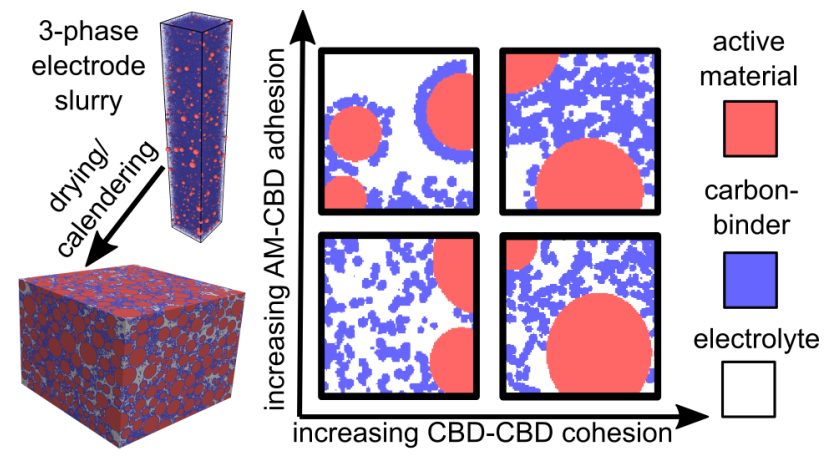

Figure 7: Graphical TOC Entry 